\title{
Global reactivity Indices and Electron Localization Function calculations in the formation of boron nitride molecule
}

\author{
Ahmed El Hadki *, Rabia Tazi, Hamza El Hadki, Najia Komiha, Abdellah Zrineh and \\ Oum Keltoum Kabbaj \\ Laboratory of Spectroscopy, Molecular Modeling, Materials, and Environment, Faculty of Sciences, \\ University Mohammed V, Rabat, Morocco
}

\begin{abstract}
The present study focuses on topological analysis of electron density population, structural and thermodynamic properties involved in the reaction between Boric Acid $\left(\mathrm{H}_{3} \mathrm{BO}_{3}\right)$ and Ammonia $\left(\mathrm{NH}_{3}\right)$ in the synthesis of boron nitride (BN) used in cancer therapy medication and biomedical applications. The compound above has similar properties to carbonaceous materials. Indeed, it exists mainly in the two cubic and hexagonal forms, which are respectively identical to diamond and graphene surfaces. DFT/ M06-2X/aug-cc-pVDZ calculations were performed to determine global reactivity indices and the reaction process that operates via three transition states. ELF function has been achieved to describe the evolution of forming bonds along the IRC path.
\end{abstract}

Keywords: Density functional theory; ELF; Interaction mechanism; Boron Nitride.

\section{Introduction}

The search for new materials with specific physical and chemical properties is a major challenge for today's industry. Regardless of the fields of application considered (emerging materials, microelectronics, environment, biomaterials, energy...), the quality of the substrate and the method of preparation are two important parameters that must be skillfully combined.

Composed of equal numbers of boron (B) and nitrogen $(\mathrm{N})$ atoms, Boron nitrides $(\mathrm{BN})$ is a twodimensional chemical compound with a layer structure. Analogous to graphite, $\mathrm{B}$ and $\mathrm{N}$ atoms are bound via strong $\mathrm{B}-\mathrm{N}$ covalent bonds, whereas the

$$
\mathrm{H}_{3} \mathrm{BO}_{3}+\mathrm{NH}_{3} \longrightarrow \mathrm{BN}+3 \mathrm{H}_{2} \mathrm{O}
$$

In this sense, we investigated the reactional pathway leading to the formation of boron nitride using density functional theory (DFT). The mechanism of the reaction process was simulated to find the intermediates, the transitions states, and the activation energies for the different steps as well as the global reactivity descriptors to examine chemical reactivity and site selectivity. Also, a topological analysis based on the electron localization function (ELF) was conducted to assess the chemical bond by determining the electronic density evolving during the reaction.

\section{Computational methods}

All DFT calculations ${ }^{7,8}$ on $\mathrm{H}_{3} \mathrm{BO}_{3}, \mathrm{BN}, \mathrm{H}_{2} \mathrm{O}$, and $\mathrm{NH}_{3}$

*Corresponding author: Ahmed El Hadki

Email address: el.hadki.ahmed@gmail.com

DOI: http://dx.doi.org/10.13171/mjc107020071343aeh weak Van der Waals forces are holding 2D layers together. BN materials exist in various allotropic forms as cubic, wurtzite, and hexagonal, depending on the hybridization between atoms.

Although its structural analogy with graphite, boron nitride exhibits wholly different and improved physical properties ${ }^{1-4}$, which renders this material as a unique and significant inorganic candidate for applications in fields of high technology, metallurgy, military industry, building materials, aerospace and electronics ${ }^{5}$.

In this paper, we present some new theoretical results concerning the reaction between boric acid and ammonia reported in literature ${ }^{6}$ expressed as follow:

were performed in the gas phase using Gaussian 09 package ${ }^{9}$ at M06-2X ${ }^{10}$ functional coupled to the augcc-pVDZ $((9 \mathrm{~s} 4 \mathrm{pld}) /[3 \mathrm{~s} 2 \mathrm{p} 1 \mathrm{~d}]) \quad$ basis $\quad$ set ${ }^{11}$ and visualized by GaussView5.0 ${ }^{12}$. The process governing this reaction was achieved by a complete optimization of the reactants, products, intermediates, and transition states without any constraint of symmetry. The latter, characterized as stationary points with one imaginary frequency, are localized on the potential energy surface and verified by an analysis of the intrinsic reaction coordinates (IRC) ${ }^{13}$. The study of the various qualitative chemical concepts such as electronic chemical potential ${ }^{14}$, chemical hardness ${ }^{15}$, electrophilicity ${ }^{16}$, and energy gap was 
conducted by density functional theory expressed, respectively, as follow:

$$
\begin{aligned}
& \mu=-\frac{1}{2}(I+A) \\
& \eta=(\mathrm{I}-\mathrm{A}) \\
& \omega=\frac{\mu^{2}}{2 \eta} \\
& \mathrm{E}_{g}=E_{\text {HOMO }}-E_{L U M O}
\end{aligned}
$$

Furthermore, the natural bond orbital analysis is employed to evaluate the charge transfer interaction ${ }^{17}$, and the contour plot for visualization results are constructed with NBO view software (version 1.1) ${ }^{18}$.

\section{Results and discussion}

The present study will be structured in four sections. Firstly, we performed an analysis of the conceptual
DFT reactivity index ${ }^{7,8}$ to explain the reactivity based on the electronic properties of the reactants involved in the reaction studied here. Secondly, natural charges are determined for boric acid to identify the electrophilic center. In the third section, IRC profile analysis was performed to verify that the transition states connect the reagents and reaction products. Afterward, a mechanistic study has been completed, and the dissociation/formation of sigma bonds along the reaction path is characterized through ELF topological analysis study.

\subsection{Global reactivity index}

The global reactivity index characterized via the conceptual DFT are practical, theoretical tools to explain the reactivity ${ }^{19,20}$. In Table 1 , we reported the energies of the frontier molecular orbitals, their difference $\left(E_{\mathrm{g}}\right)$ as well as the global reactivity descriptors for the two reagents.

Table 1. $\mathrm{E}_{\text {номо, }} \mathrm{E}_{\mathrm{Lumo}}$, and $\mathrm{E}_{\mathrm{g}}$ in a.u. Electronic chemical potential $\mu$, chemical hardness $\eta$, global electrophilicity $\omega$ in $\mathrm{eV}$ at M06-2X/aug-cc-pVDZ level of theory.

\begin{tabular}{|c|c|c|c|c|c|c|}
\hline Reactants & $\mathrm{E}_{\text {HOMO }}$ & $\mathrm{E}_{\mathrm{LUMO}}$ & $\mathrm{E}_{\mathrm{g}}$ & $\mu$ & $\eta$ & $\omega$ \\
\hline $\mathrm{B}(\mathrm{OH})_{3}$ & -0.406 & -0.010 & 0.397 & -5.660 & 10.803 & 1.497 \\
\hline $\mathrm{NH}_{3}$ & -0.338 & -0.005 & 0.333 & -4.680 & 9.061 & 1.197 \\
\hline
\end{tabular}

We notice that $\mathrm{B}(\mathrm{OH})_{3}$ has a chemical potential lower, a higher electrophilicity index, so the electronic transfer is made from $\mathrm{NH}_{3}$ to $\mathrm{B}(\mathrm{OH})_{3}$ inducing that the $\mathrm{B}(\mathrm{OH})_{3}$ will play the role of the electrophile.

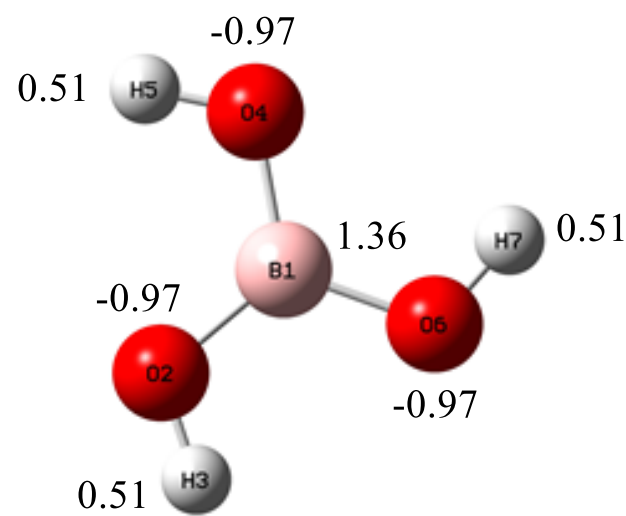

\subsection{Natural charges}

The calculation of the electronic population on each atom of the reactants allows us to determine the most favorable site for attack. The values obtained are reported in Figure 1.

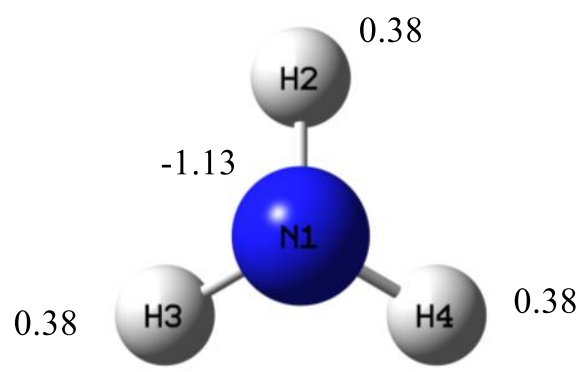

Figure 1. Electronic population of the atom in the neutral $\mathrm{B}(\mathrm{OH})_{3}$ and $\mathrm{NH}_{3}$

The calculation of the natural charges on the neutral molecule $\mathrm{B}(\mathrm{OH})_{3}$ and $\mathrm{NH}_{3}$ shows that the most electropositive center is the boron atom (1.36) and the most electronegative center is the nitrogen atom $(-1.13)$. Therefore, the reaction will take place between these two sites.

\subsection{Mechanistic study}

First, we located three transition states on the potential energy surface using the QST2 method, and we verified that only one imaginary frequency is obtained. In a second step, we performed IRC calculations to determine the possible presence of local intermediates along with the reaction profile as well as to verify that the first transition state found was connected to the reagents on one side and a local minimum on the other. The second transition state is associated with both sides to a reactional intermediate, whereas the last leads to the final product. Figure 2 and Table 2 show, respectively, the curve corresponding to the evolution of the energy $\mathrm{E}$ as a function of the intrinsic reaction coordinates: $\mathrm{E}=\mathrm{f}(\mathrm{RC})$ and the relative energies values in gasphase. 


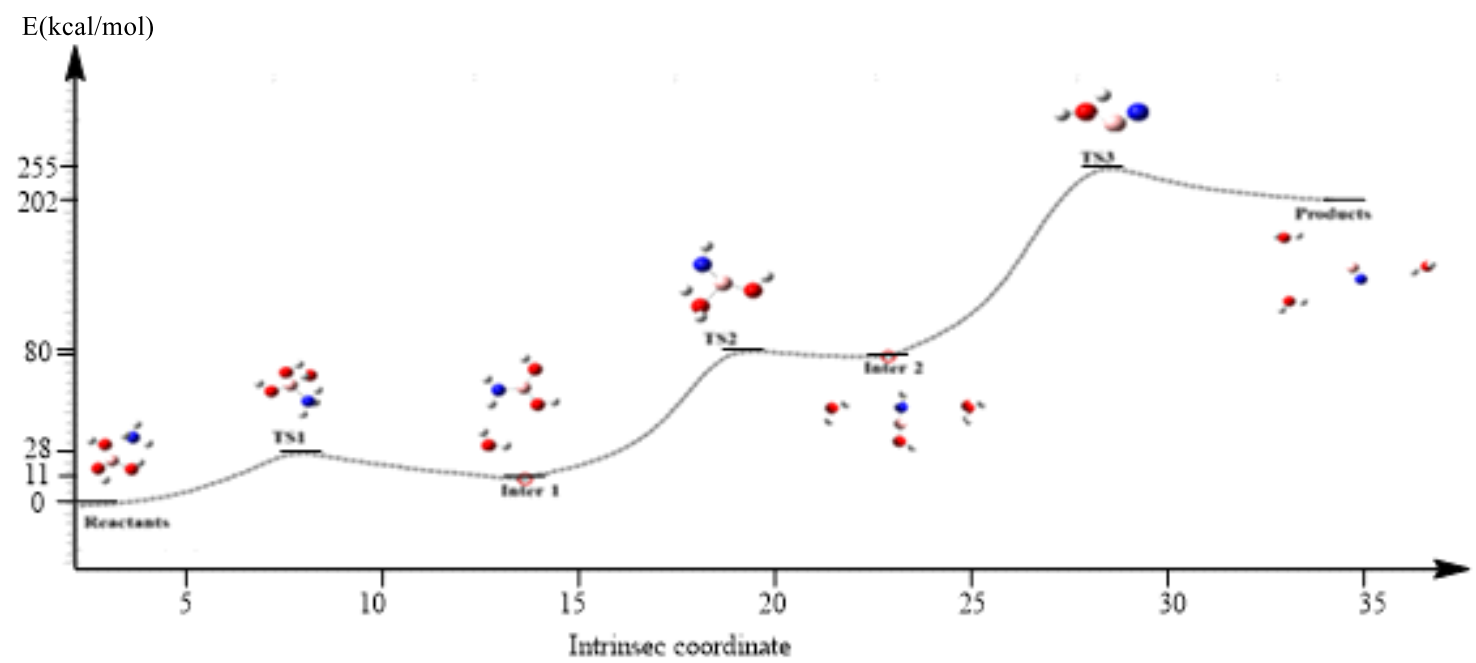

Figure 2. Relative energies of transition structures, intermediates, and products

The energy profile involved in the mechanism of this complex reaction highlights three transition states and two intermediates. The nature of each stationary point has been established by a vibrational frequency calculation, which has shown that the minima have positive frequencies. The three transitions states are characterized by the presence of a single imaginary frequency having respective values of $1278 \mathrm{i}, 780 \mathrm{i}$, and $1492 \mathrm{i}$.

Table 2. Total energies $\mathrm{E}$ (a.u) and relative energies $\Delta \mathrm{E}(\mathrm{kcal} / \mathrm{mol})$ of stationary points.

\begin{tabular}{|c|c|c|}
\hline & E (a.u) & $\Delta \mathbf{E}(\mathbf{k c a l} / \mathbf{m o l})$ \\
\hline Reactants & -308.991 & - \\
\hline TS1 & -308.947 & 27.808 \\
\hline Inter 1 & -308.974 & 10.588 \\
\hline TS2 & -308.864 & 79.560 \\
\hline Inter 2 & -308.859 & 82.541 \\
\hline TS3 & -308.668 & 202.318 \\
\hline Products & -308.584 & 255.038 \\
\hline
\end{tabular}

Analysis of the IRC data indicates that the studied reaction follows a three-step, three-phase mechanism. The first phase starts with the disruption of one of the $\mathrm{N}-\mathrm{H}$ bonds of the ammonia group, followed by the migration of the hydrogen atom corresponding to the transition state TS1. Thus, forming an O-H bond with one of the hydroxyl group leading to the disruption of a $\mathrm{B}-\mathrm{OH}_{2}$ bond and formation of a water molecule and the first reaction intermediate $\mathrm{H}_{4} \mathrm{BNO}_{2}$. The same process takes place for the departure of the other two water molecules via the formation of a second reaction intermediate leading to the final $\mathrm{BN}$ product.

The analysis of previous data shows three transitions state on the reactional path. The activation barriers associated with the TS1, TS2, and TS3 are respectively $27.8,79.6,202.3 \mathrm{kcal} / \mathrm{mol}$. The formation of the products is endothermic since the energy involved in the reaction is positive $(202.3 \mathrm{kcal} / \mathrm{mol})$ and will most likely require a significant energy input as experimentally predicted ${ }^{6}$.

Subsequently, a topological analysis of the electronic localization function (ELF) was carried out to study the evolution of the electron density during the approach of the 2 reagents.

\subsection{ELF analysis of electronic structures}

Based on the optimized structures for both reagents, a topological ELF analysis of the valence attractors was performed at the DFT/M06-2X/aug-cc-pVDZ level. The following figure shows the main mono and disynaptic basins characterized during this analysis. The values of the electronic population relating to the valence basins are grouped in Figure 3 and Table 3. 


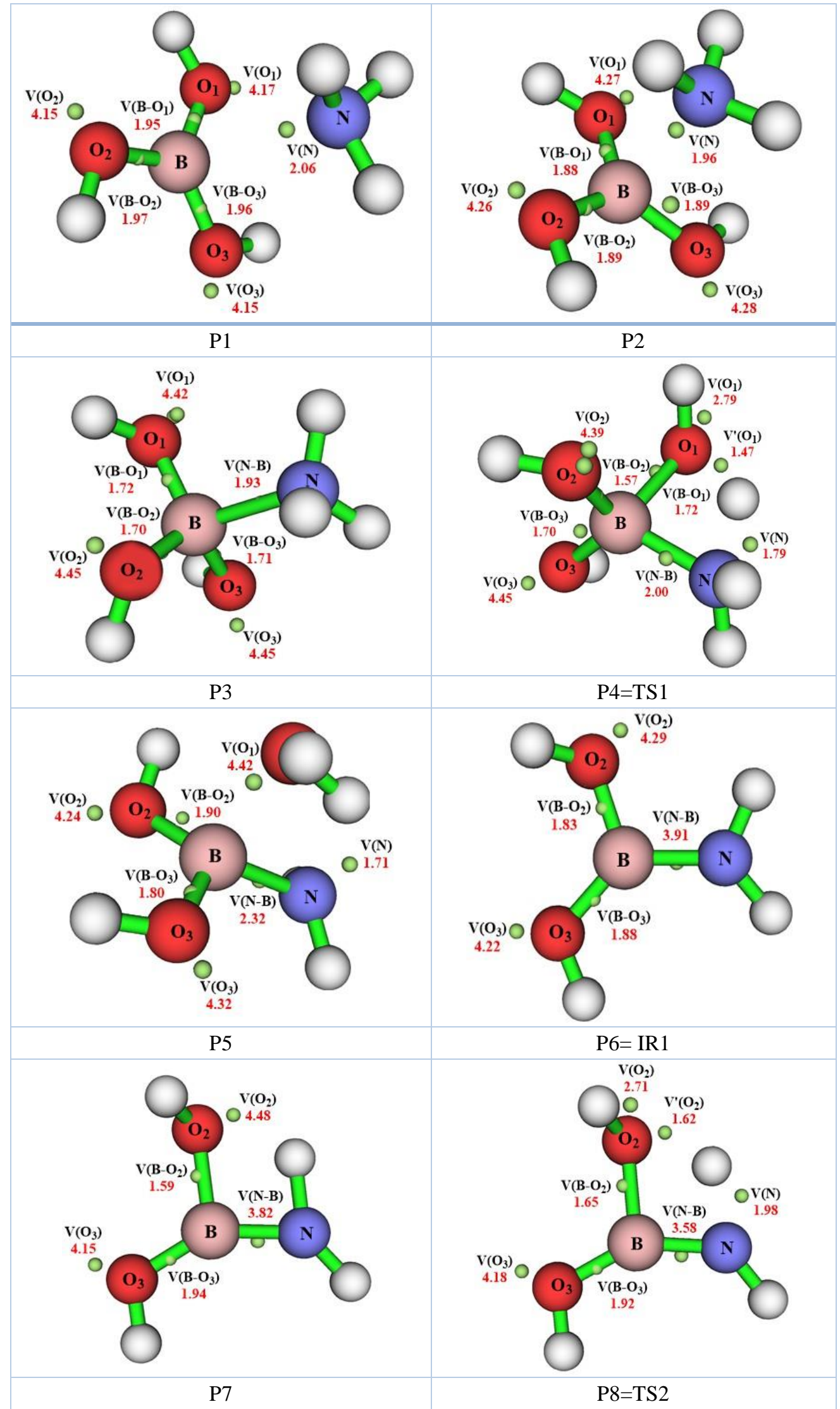




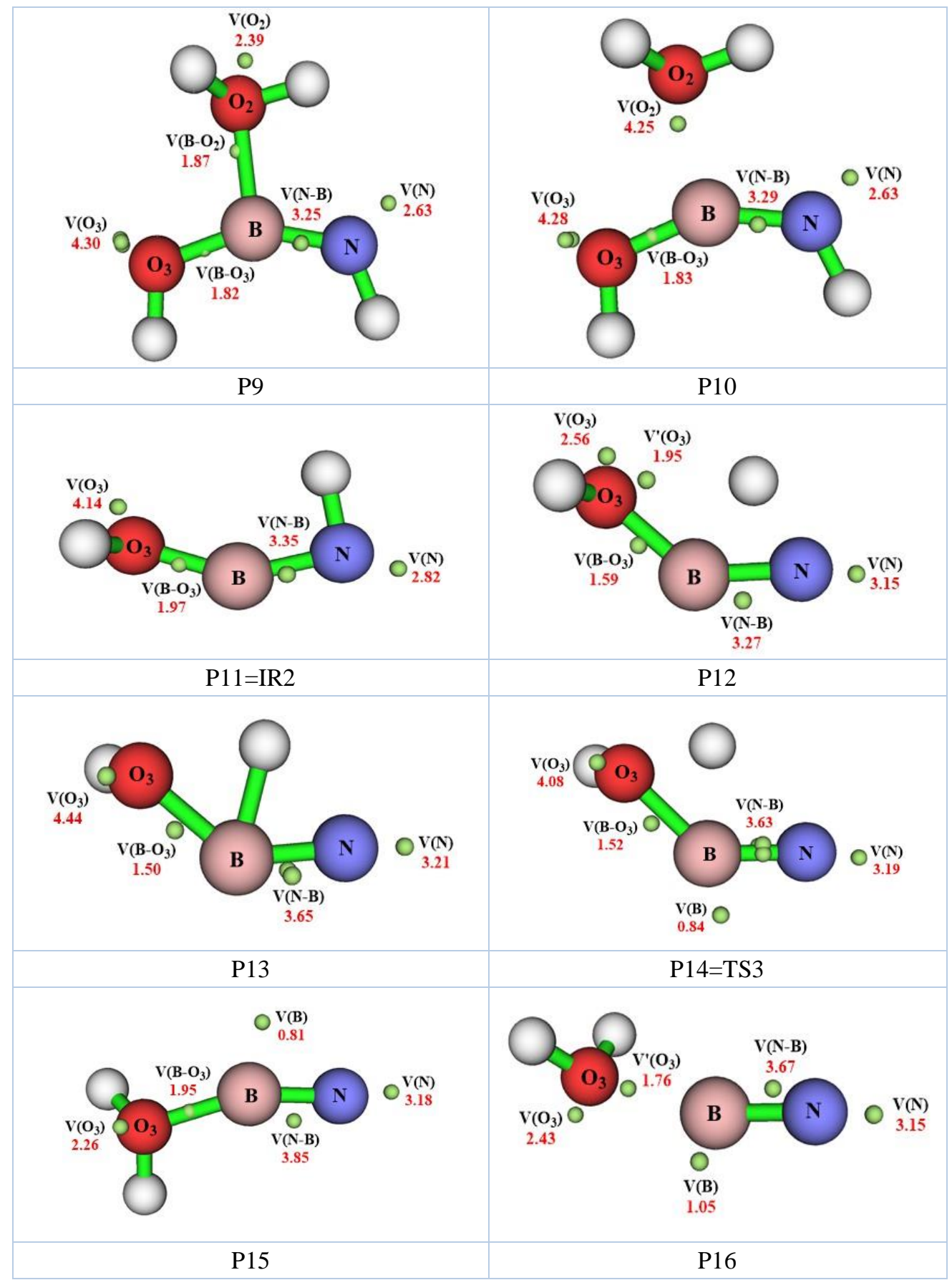

Figure 3. Monosynaptic and disynaptic basins of the $\mathrm{H}_{3} \mathrm{BO}_{3}-\mathrm{NH}_{3}$ complex

Table 3. ELF Valence basin populations of chosen points along the IRC path in e.

\begin{tabular}{|c|c|c|c|c|c|c|c|c|c|c|c|c|c|c|c|c|}
\hline & P1 & P2 & P3 & $\begin{array}{c}\text { P4 } \\
\text { (TS1) }\end{array}$ & P5 & $\begin{array}{c}\text { P6 } \\
\text { (IR1) }\end{array}$ & P7 & $\begin{array}{c}\text { P8 } \\
\text { (TS2) }\end{array}$ & P9 & P10 & $\begin{array}{c}\text { P11 } \\
\text { (IR2) }\end{array}$ & P12 & P13 & $\begin{array}{c}\text { P14 } \\
\text { (TS3) }\end{array}$ & P15 & P16 \\
\hline $\mathrm{V}(\mathrm{N})$ & 2.1 & 2 & - & 1.8 & 1.7 & - & - & 2 & 2.6 & 2.6 & 2.8 & 3.2 & 3.2 & 3.2 & 3.2 & 3.2 \\
\hline $\mathrm{V}(\mathrm{O} 1)$ & 4.2 & 4.3 & 4.4 & 2.8 & 4.4 & - & - & - & - & - & - & - & - & - & - & - \\
\hline $\mathrm{V}^{\prime}(\mathrm{O} 1)$ & - & - & - & 1.5 & - & - & - & - & - & - & - & - & - & - & - & - \\
\hline $\mathrm{V}(\mathrm{O} 2)$ & 4.2 & 4.3 & 4.5 & 4.4 & 4.2 & 4.3 & 4.5 & 2.7 & 2.4 & 4.3 & - & - & - & - & - & - \\
\hline $\mathrm{V}^{\prime}(\mathrm{O} 2)$ & - & - & - & - & - & - & - & 1.6 & - & - & - & - & - & - & - & - \\
\hline $\mathrm{V}(\mathrm{O} 3)$ & 4.2 & 4.3 & 4.5 & 4.5 & 4.3 & 4.2 & 4.2 & 4.2 & 4.3 & 4.3 & 4.1 & 2.6 & 4.4 & 4.1 & 2.3 & 2.4 \\
\hline $\mathrm{V}^{\prime}(\mathrm{O} 3)$ & - & - & - & - & - & - & - & - & - & - & - & 2 & - & - & - & 1.8 \\
\hline
\end{tabular}




\begin{tabular}{|c|c|c|c|c|c|c|c|c|c|c|c|c|c|c|c|c|}
\hline $\mathrm{V}(\mathrm{B})$ & - & - & - & - & - & - & - & - & - & - & - & - & - & 0.8 & 0.8 & 1.1 \\
\hline $\mathrm{V}(\mathrm{B}-\mathrm{O} 1)$ & 2 & 1.9 & 1.7 & 1.7 & - & - & - & - & - & - & - & - & - & - & - & - \\
\hline $\mathrm{V}(\mathrm{B}-\mathrm{O} 2)$ & 2 & 1.9 & 1.7 & 1.6 & 1.9 & 1.8 & 1.6 & 1.7 & 1.9 & - & - & - & - & - & - & - \\
\hline $\mathrm{V}(\mathrm{B}-\mathrm{O} 3)$ & 2 & 1.9 & 1.7 & 1.7 & 4.3 & 1.9 & 1.9 & 1.9 & 1.8 & 1.8 & 2 & 1.6 & 1.5 & 1.5 & 2 & - \\
\hline $\mathrm{V}(\mathrm{N}-\mathrm{B})$ & - & - & 1.9 & 2 & 2.3 & 3.9 & 3.8 & 3.6 & 3.3 & 3.3 & 3.4 & 3.3 & 3.7 & 3.6 & 3.9 & 3.7 \\
\hline
\end{tabular}

The results reported above show that at points P1 and $\mathrm{P} 2$, we can observe the presence of a monosynaptic basin on the nitrogen atom of the $\mathrm{NH}_{3}$ group allowing the possible formation of a dative bond between the two fragments. At point P4, the first transition state on the potential surface, the formation of the $\mathrm{BN}$ bond and the departure of hydrogen leading to the appearance of two new monosynaptic pools $\mathrm{V}(\mathrm{N})$ and $\mathrm{V}^{\prime}(\mathrm{O} 1)$ on the nitrogen and oxygen atoms of the $\mathrm{H}_{6} \mathrm{BNO}_{3}$ complex with a population of $1.79 \mathrm{e}$ and 1.47 e respectively. At point 5 , the monosynaptic basin $\mathrm{V}(\mathrm{O} 1)$ disappears following the establishment of the $\mathrm{OH}$ bond inducing the formation and followed by the departure of the first water molecule. From point 6 , the process leading to the formation and the departure of the water molecule is renewed until the formation of the compound $\mathrm{BN}$.

\section{Conclusion}

We established, using the global descriptor of reactivity, that the electronic transfer will take place from the ammonia to the boric acid. Based on the NPA charge, the oxygen atoms of hydroxyl groups are the favorable centers for the nucleophilic attack-inducing eventual formation of water molecules. The IRC profile reveals an endothermic mechanism with three phases and three steps. The ELF topological analysis confirms that molecular mechanism occurs via a three stages three-steps for this reaction.

\section{References}

1- C. Zhi, Y. Bando, C. Tang, H. Kuwahara, D. Golberg, Large-Scale Fabrication of Boron Nitride Nanosheets and Their Utilization in Polymeric Composites with Improved Thermal and Mechanical Properties, Adv Mater., 2009, 21, 2889-2893.

2- J. Taha-Tijerina, T. N. Narayanan, G. Gao, M. Rohde, D. A. Tsentalovich, M. Pasquali, P. M. Ajayan, Electrically Insulating Thermal Nano-Oils Using 2D Fillers, ACS Nano., 2012, 6, 1214-1220.

3- H. Zhu, Y. Li, Z. Fang, J. Xu, F. Cao, J. Wan, C. Preston, B. Yang, L. Hu, Highly Thermally Conductive Papers with Percolative Layered Boron Nitride Nanosheets, ACS Nano., 2014, 8, 3606-3613.

4- L. H. Li, J. Cervenka, K. Watanabe, T. Taniguchi, Y. Chen, Strong Oxidation Resistance of Atomically Thin Boron Nitride Nanosheets, ACS Nano., 2014, 8, 1457-1462.
5- Z. Liu, Y. Gong, W. Zhou, L. Ma, J. Yu, J. C. Idrobo, J. Jung, A. H. MacDonald, R. Vajtai, J. Lou, P. M. Ajayan, Ultrathin high-temperature oxidation-resistant coatings of hexagonal boron nitride, Nat Commun., 2013, 4, 2541.

6- B. Singh, G. Kaur, P. Singh, K. Singh, B. Kumar, A. Vij, M. Kumar, R. Bala, R. Meena, A. Singh, A. Thakur, A. Kumar, Nanostructured Boron Nitride with High Water Dispersibility For Boron Neutron Capture Therapy, Sci Rep., 2016, 6, 35535.

7- W. Koch, M. C. Holthausen, A Chemist's Guide to Density Functional Theory, 2nd, 2001.

8- R. G. Parr, Density Functional Theory of Atoms and Molecules, Horizons of Quantum Chemistry, Springer Netherlands: Dordrecht, 1980, 5-15.

9- M. J. Frisch, G. W. Trucks, H. B. Schlegel, G. E. Scuseria, M. A. Robb, J. R. Cheeseman, G. Scalmani, V. Barone, G. A. Petersson, H. Nakatsuji, X. Li, M. Caricato, A. Marenich, J. Bloino, B. G. Janesko, R. Gomperts, B. Mennucci, H. P. Hratchian, J. V. Ortiz, A. F. Izmaylov, J. L. Sonnenberg, D. Williams Young, F. Ding, F. Lipparini, F. Egidi, J. Goings, B. Peng, A. Petrone, T. Henderson, D. Ranasinghe, V. G. Zakrzewski, J. Gao, N. Rega, G. Zheng, W. Liang, M. Hada, M. Ehara, K. Toyota, R. Fukuda, J. Hasegawa, M. Ishida, T. Nakajima, Y. Honda, O. Kitao, H. Nakai, T. Vreven, K. Throssell, J. A. Montgomery, Jr., J. E. Peralta, F. Ogliaro, M. Bearpark, J. J. Heyd, E. Brothers, K. N. Kudin, V. N. Staroverov, T. Keith, R. Kobayashi, J. Normand, K. Raghavachari, A. Rendell, J. C. Burant, S. S. Iyengar, J. Tomasi, M. Cossi, J. M. Millam, M. Klene, C. Adamo, R. Cammi, J. W. Ochterski, R. L. Martin, K. Morokuma, O. Farkas, J. B. Foresman, D. J. Fox, Gaussian 09, Gaussian Inc, Wallingford CT, 2009.

10-Y. Zhao, D. G. Truhlar, The M06 suite of density functionals for main group thermochemistry, thermochemical kinetics, noncovalent interactions, excited states, and transition elements: two new functionals and systematic testing of four M06-class functionals and 12 other functionals, Theor Chem Account., 2008, 120, 215-241.

11-T. H. Dunning, Gaussian basis sets for use in correlated molecular calculations. I. The atoms boron through neon and hydrogen, J Chem Phys., 1989, 90, 1007-1023. 
12-R. Dennington, T.A. Keith, J.M. Millam, Gauss view, Version 5, Semichem. Inc., Shawnee Mission, 2009.

13-C. Gonzalez, H.B. Schlegel. An improved algorithm for reaction path following, J. Chem.Phys., 1989, 9, 2154-2161.

14-S. Saha, R. K. Roy, S. Pal, CDASE-A reliable scheme to explain the reactivity sequence between Diels-Alder pairs, Phys Chem Phys., 2010, 12, 9328.

15-P. Geerlings, F. De Proft, W. Langenaeker, Conceptual Density Functional Theory, Chem Rev., 2003, 103, 1793-1874.

16-T. Joseph, H. T. Varghese, C. Y. Panicker, K. Viswanathan, M. Dolezal, C. Van Alsenoy, Spectroscopic (FT-IR, FT-Raman), first-order hyperpolarizability, NBO analysis, $\mathrm{HOMO}$ and LUMO analysis of $\mathrm{N}-[(4-$

(trifluoromethyl)phenyl]pyrazine-2-carboxamide by density functional methods, Arabian Journal of Chemistry, 2017, 10, S2281-S2294.
17-Z. Hasanzade, H. Raissi, Solvent/co-solvent effects on the electronic properties and adsorption mechanism of anticancer drug Thioguanine on Graphene oxide surface as a nanocarrier: Density functional theory investigation and a molecular dynamic, Applied Surface Science, 2017, 422, 1030-1041.

18-E. D. Glendening, A. E. Reed, J. E. Carpenter, F. Weinhold, NBO Programm, Version 1.1.

19-H. El Hadki, F. Hlimi, M. Salah, K. Marakchi, N. Komiha, O. K. Kabbaj, Theoretical Study of Reaction Between Nitrilimine and 1,4 Oxazine 2 Carboxylate by MP2 and DFT Methods, Oriental Journal of Chemistry, 2018, 34, 2992-2997.

20-R. Tazi, H. E. Hadki, M. Salah, A. Zrineh, M. E. Azzouzi, N. Komiha, Theoretical Approach of the Adsorption of Herbicide Amitrole on the Soil using DFT Method, Oriental Journal of Chemistry, 2018, 34, 1240-1248. 